\title{
Comparative statics in Cournot free entry equilibrium
}

\author{
Luis C. Corchón ${ }^{a}, *$, Isabel Fradera ${ }^{\mathrm{b}}$ \\ a Department of Economics, Universidad Carlos III, c/ Madrid 126, Getafe 28903, Madrid, Spain \\ ${ }^{\mathrm{b}}$ Department of Economics, Universidad Autónoma de Barcelona, Edifici B, Bellaterra, 08193 Barcelona, \\ Spain
}

Received 31 August 1998; received in revised form 31 March 2002; accepted 30 April 2002

\begin{abstract}
In this paper we study the effects of a change in an exogenous variable (the fixed cost or a parameter in the demand function) on the output and the number of active firms in a Symmetric Cournot Equilibrium with Free Entry (SCEFE). The results obtained here are different from those obtained in the Cournot model with a given number of firms. In particular, an increase in demand might yield a decrease in the output of the industry. We also show that any observation on prices, profits and number of firms is compatible with the assumption that the market is in a SCEFE. If fixed costs can be observed, there is a loose relationship between the profit rate and the number of active firms. This result is used as a warning against the use of the profit rate as a measure of an anticompetitive position and against the Structure-Conduct-Performance paradigm.
\end{abstract}

Keywords: Entry; Comparative statics; Rate of profits; Anti-trust policy

JEL classification: L13

\section{Introduction}

A large number of papers have analyzed comparative static under imperfect competition. In the case of the Cournot model with a given number of firms the list includes McManus (1962, 1964), Frank (1965), Ruffin (1971), Okuguchi (1973), Seade (1980), Szidarovsky and Yakowitz (1982), Dixit (1986), Quirmbach (1988) and Corchon (1994). All these papers assume a strong concavity condition which implies

*Corresponding author Tel : +34-91-624-9617; fax: +34-91-624-9329

E-mail address: lcorchon@eco uc3m es (L C Corchón) 
that the best reply of any firm is a decreasing function of the output of other firms. The latter property has been called by Bulow et al. (1985) strategic substitution. Comparative statics in the case of strategic complementarities (best replies are increasing, like in price competition with, say, linear demand) was worked out by Lippman et al. (1987), Vives (1990), Milgrom and Roberts (1990) and Milgrom and Shannon (1992).

The existence of a Cournot equilibrium with free entry was first considered by Novshek (1980). However, to the best of our knowledge, no attention has been paid to the properties of this kind of equilibrium. Our paper attempts to remedy this situation.

In Section 2 we present the model. We assume that all firms are identical, strong concavity holds for positive output and fixed costs are positive. The only decision for firms is the quantity of output (zero output corresponds to no entry). The strategic behavior of a firm is described by a mapping which is continuous except at the entry-exit point. From this it follows easily that a Symmetric Cournot Equilibrium with Free Entry (SCEFE in the sequel) exists.

In Section 3 we study comparative statics. We show that a change in the fixed cost has the effect that intuition suggests: the number of active firms and aggregate output move in opposite direction of the fixed cost while individual output moves in the same direction. However, the effect of a change in demand or marginal costs is, in general, ambiguous. We obtain definitive predictions only when an additional and very restrictive assumption holds (namely Assumption 6, see the main text). We also provide an example in which an increase in demand decreases the number of active firms and aggregate output. Thus, our main conclusion in this part of the paper is that there is an important difference between the Cournot model with and without entry. In the latter, under strong concavity, aggregate output changes monotonically with marginal costs or with the parameters of the demand function (see, e.g., Corchon, 1994), Proposition 6). In the case of free entry, strong concavity is not enough to yield definitive predictions on aggregate output.

In Section 4 we study the observational implications of our model both at individual and market level. Firstly, we assume that the behavior of the representative firm can be perfectly observed (i.e., it can be replicated in a laboratory). This behavior relates the aggregate output of the competitors of the representative firm (denoted by $Q$ ) and the output of the representative firm (denoted by $q$ ). We derive necessary and sufficient conditions for a set of data on $q$ and $Q$ to be rationalized as the behavior of a profit maximizing firm. These conditions are: (1) for strictly positive values of $q, q$ must be strictly decreasing on $Q$ with slope larger than minus one; (2) there is an unique value of $Q$ (say $y$ ) for which $q$ is either zero or positive; (3) for $Q$ larger than $y, q$ is always zero. Secondly we consider market behavior. Suppose that an observation of market price, profits and number of firms can be obtained from an experiment or from a real market (notice that in this case, profits are observable). The question is: What triples \{price, profits, number of active firms\} can be generated as SCEFE? In order to tie our hands as tight as possible we assume strong restrictions on the form of both demand (assumed to be of unit elasticity) and marginal cost (assumed to be constant). However, despite such strong restrictions, any triple \{price, profits, number of active firms can be generated as SCEFE. If fixed costs are also observable, there is a loose relationship between the 
number of active firms and the (extraordinary) profit rate. ${ }^{1}$ In particular if $\tilde{\pi}$ are observed profits, $\tilde{k}$ the observed fixed cost and $\tilde{n}$ is the observed number of active firms, it must be that

$$
\frac{\tilde{\pi}}{\tilde{k}}<\frac{1}{\tilde{n}^{2}}+\frac{2}{\tilde{n}} .
$$

This implies that with 20 active firms (a number of firms that many economist would consider large), the upper bound on the profit rate is, approximately, 10\%, larger than most actual profit rates reported in Fortune. Thus the main conclusion of this part of the paper is that the Cournot model with free entry does not support the view that there is a direct relationship between profitability $(\tilde{\pi} / \tilde{k})$ and concentration $(1 / \tilde{n})$. This has important consequences on, at least, three counts:

(a) Antitrust policy. A high profit rate does not necessarily imply lack of competition and/or collusion among existing firms. This has been pointed out by several authors (see, e.g., Cabral, 2000, p. 157; and Martin, 1993, pp. 499-507). They have noticed that profitability might be a poor proxy for the price/marginal cost ratio, which is the right measure of an anticompetitive position. Our results make this point sharply by showing that even if information about market conditions is available, the only implication of (Cournot) competition and free entry is that there is an upper bound on the rate of profit and that this bound may be very large.

(b) Structure-Conduct-Performance paradigm. According to Bain "We would anticipate some complex relationship of, at least, three variables, profit rate, degree of seller concentration and conditions of entry" (Bain, 1956, p. 191). If entry conditions are summarized by fixed costs, this relationship is precisely the inequality above. Our theoretical result could provide a possible explanation of why the relationship postulated by Bain is "weak ... often ... not statistically significant" (Carlton and Perloff, 1990, p. $375)$.

(c) Entry and rates of profit. There are persistent differences in the rate of profits across industries. Moreover, high profit rates often decline slowly in highly concentrated industries (see, e.g., Carlton and Perloff, 1990, pp. 372, 381). Again, our result, provides a possible explanation of this fact, because it shows that free entry is compatible with different profit rates, even if the functional form of demand and cost functions is the same in all markets.

\section{The model}

In this section we present our main definitions and some basic results. There are countably many identical potential firms. Let $n$ be the number of active firms, $q$ be the

\footnotetext{
${ }^{1}$ Recall that these are extraordinary profits, i e , profits net of opportunity costs

${ }^{2}$ Profit rates reported in the magazine Fortune usually vary between 4 and $7 \%$ Subtracting the interest rate we obtain the rate of extraordinary profits Thus if the interest rate is assumed to be $4 \%$ the rates reported there correspond to rates of extraordinary profits between 0 and $3 \%$
} 
output of a representative firm and $Q$ be the aggregate output of the competitors of the representative firm. Let $k$ be the fixed cost and $K$ the space of all possible fixed costs. Let $b$ be a parameter in the demand or the variable cost functions (i.e., subsidies, income, other prices, etc.) and $B$ the set of all possible $b$ 's. Let $B$ and $K$ be subsets of $\mathbb{R}_{+}$. The payoff function of the representative firm, denoted by $\pi: \mathbb{R}_{+}^{2} \times B \times K \rightarrow \mathbb{R}$ is such that:

$$
\begin{aligned}
& \pi(q, Q, b, k)=V(q, Q, b)-k \text { if } q>0 \\
& \pi(q, Q, b, k)=0 \text { if } q=0
\end{aligned}
$$

where $V$ is a twice differentiable function into $\mathbb{R}_{+}$. Partial derivatives of $V$ will be denoted by a subscript, i.e., $V_{q}, V_{Q}, V_{b}$, etc. It is implicit that we are assuming product homogeneity since only the sum of outputs of competitors $(Q)$ is payoff relevant. However, product heterogeneity of a certain kind can be considered as well (see, e.g., Yarrow 1985, p. 517).

The best reply correspondence, denoted by $R($ ), is defined as follows:

$$
R(Q, b, k)=\arg \max _{q} \pi(q, Q, b, k) .
$$

And the best reply correspondence under zero fixed costs is,

$$
r(Q, b)=\arg \max _{q} V(q, Q, b) .
$$

The following assumptions will be maintained throughout the paper:

A.1. $0 \notin K$. $\forall(b, k) \in B \times K, \max _{q} V(q, 0, b)>k$.

A.2. There is $\bar{q}$ such that $\forall Q, V(q, Q, b) \leq 0$ if $q \geq \bar{q}$.

A.3. There is $\bar{Q}$ such that $\forall q, V(q, Q, b) \leq 0$ if $Q \geq \bar{Q}$.

A.4 $V_{Q}<0$ on the interior of $\mathbb{R}_{+}^{2} \times B$.

A.5 $V_{q q}<V_{q Q}<0$ on the interior of $\mathbb{R}_{+}^{2} \times B$.

These assumptions describe several properties of the payoff function. A.1 postulates a positive fixed cost, which can be covered in case of a monopoly. Under A.2 no firm will produce an output larger than $\bar{q}$ since $\pi(q, Q, b, K)<0, \forall q \geq \bar{q}$. A.3 implies that if the output of the competitors exceed $\bar{Q}$, the representative firm cannot make positive payoffs. In the case in which payoffs are profits, A.4 is equivalent to say that the demand function is strictly decreasing. Finally A.5 implies three things: (a) $q$ and $Q$ are strategic substitutes (since $\mathrm{d} R / \mathrm{d} Q=-V_{q Q} / V_{q q}<0$ whenever $R$ is differentiable). (b) The best reply function is a contraction (since $|\mathrm{d} R / \mathrm{d} Q|<1$ ). (c) $V$ is strictly concave on $q$. In the case in which payoffs are profits, A.5 implies a standard assumption in the literature on Cournot equilibrium (see e.g., Hahn, 1962; and Friedman, 1982, p. 496, Assumption 3), namely

$$
q p_{Q Q}+p_{Q}<0 \text { and } p_{Q}-C_{q q}<0
$$

where $p$ is the inverse demand function and $C$ is the cost function of the representative firm. The strong concavity condition mentioned before corresponds to the above assumption in the case of a general payoff function. We now present the main definition of the paper. 
Definition 1. A Symmetric Cournot Equilibrium with Free Entry (SCEFE) for $(b, k)$ is a pair $\left(n^{*}, q^{*}\right) \in \mathbb{N} \times \mathbb{R}_{+}$such that:

(i) $q^{*} \in R\left(\left(n^{*}-1\right) q^{*}, b, k\right)$.

(ii) $0 \in R\left(n^{*} q^{*}, b, k\right)$.

There are three aspects of our definition that are worthwhile discussing: first, in a SCEFE, entry and output setting are simultaneous. If entry and output setting are sequential, the notion of equilibrium would be that of Symmetric Subgame Perfect Cournot Equilibrium (SSPCE). Lopez-Cunat (1997) has shown that under assumptions similar to ours, the set of SSPCE is contained in the set of SCEFE and thus results similar to ours can be obtained in the sequential model. Second, we disregard mixed strategies. This is not the place to discuss the relative merits of mixed and pure strategies: we just notice that the interpretation of mixed strategies is, sometimes, troublesome and that the bulk of industrial organization has ignored mixed strategies. Thus, given that this is the first paper studying comparative statics of SCEFE, it appears that concentrating on pure strategy equilibria is a conservative but sound research strategy. See Cabral (1997) for an interesting paper on entry and mixed strategies. Finally we concentrate on symmetric equilibria in the sense that the output of active firms is identical (however, notice that in a SCEFE there are inactive firms). It is easy to see that there are no asymmetric equilibria. Let $x$ be aggregate output. For any two firms with strictly positive output, say $q_{i}$ and $q_{j}$ we must have $V_{q}\left(q_{i}, x-q_{i}, b, k\right)=V_{q}\left(q_{j}, x-\right.$ $\left.q_{j}, b, k\right)$. Because $\partial V_{q} / \partial q=V_{q q}-V_{q Q}<0$ this implies $q_{i}=q_{j}$.

The existence of a SCEFE can be easily established.

Theorem 1. There is a SCEFE for all $(b, k) \in B \times K$.

Proof. It follows from well-known arguments, see e.g., Proposition 4.8 in Corchon (1996), appendix to Chapter 4.

\section{Comparative statics}

In this section we present our results on comparative statics. Let

$$
E(b, k)=\{(n, q) /(n, q) \text { is a SCEFE for }(b, k)\} .
$$

$E(b, k)$ can contain more than one element, even though, as we remarked before, the Cournot equilibrium for given $n$ is unique. Given this lack of uniqueness we have two options. The first is to work with sets of equilibria. The second is to make a selection on the set of SCEFE such that equilibrium becomes unique. The first option has the advantage of being more comprehensive but it yields lengthy proofs. The second option has the advantage of being simpler and to yield analogous results to the first option. In this paper we follow the second route. Readers interested in the first option should consult Corchon and Fradera (1996) where we prove similar results to those presented here for the case where SCEFE may be a set. 
Among all SCEFE we will select the equilibrium with the largest number of firms. ${ }^{3}$ In this equilibrium aggregate output is maximal among all SCEFE. ${ }^{4}$ The reason for choosing this selection is discussed in the final section. Abusing notation, the SCEFE with the largest number of firms will be denoted by SCEFE as before. Let $e(b, k)$ be the selection of $E(b, k)$ such that the number of firms is the largest. We first study the effect of changes in $k$.

Theorem 2. Let $\left(n_{1}, q_{1}\right)=e\left(b, k_{1}\right)$ and $\left(n_{2}, q_{2}\right)=e\left(b, k_{2}\right)$, with $k_{1}<k_{2}$. Then, $n_{1} \geq n_{2}$, $q_{1} \leq q_{2}, n_{1} q_{1} \geq n_{2} q_{2}$.

Proof. Let $\hat{Q}(k)$ be the value of which a firm is indifferent between producing a positive output and not producing at all (see Theorem 4 below for a formal proof of the existence of $\hat{Q}(k))$. It is clear that if $k_{1}<k_{2}, \hat{Q}\left(k_{1}\right)>\hat{Q}\left(k_{2}\right)$. Other than this, the best reply correspondence if the fixed cost were $k_{1}$ and the best reply correspondence if the fixed cost were $k_{2}$ are identical because fixed costs only matter in determining if the output is positive or zero.

The SCEFE with maximal number of firms when the fixed cost is $k_{1}$ is characterized by the following:

$$
\begin{aligned}
& q_{1} \in R\left(q_{1}\left(n_{1}-1\right), b, k_{1}\right) . \\
& q \notin R\left(q\left(n_{1}+v\right), b, k_{1}\right) \forall q \in \mathbb{R}_{+}, \quad \forall v=0,1, \ldots .
\end{aligned}
$$

Now, we have two cases. If $q_{1} \in R\left(q_{1}\left(n_{1}-1\right), b, k_{2}\right), q \notin R\left(q\left(n_{1}+v\right), b, k_{2}\right) \quad \forall q \in$ $\mathbb{R}_{+}, \forall v=0,1, \ldots$ Thus, $e\left(b, k_{1}\right)=e\left(b, k_{2}\right)$ and the result follows.

If $q_{1} \notin R\left(q_{1}\left(n_{1}-1\right), b, k_{2}\right)$ then it must be that $n_{2}<n_{1}$. Since $R$ is decreasing in $Q$, $q_{1}<q_{2}$. Since $0>\mathrm{d} R / \mathrm{d} Q>-1, n_{1} q_{1}>n_{2} q_{2}$.

Theorem 2 says that a decrease in the value of the fixed cost, increases (weakly) the equilibrium number of firms, decreases (weakly) the output of the representative firm and increases (weakly) aggregate output. This agrees with our intuition and with the results obtained in the Cournot model with a given number of firms (see the Refs. given in Section 1).

Let us now turn our attention to the effects of a change in $b$. This change may have ambiguous effects because it affects simultaneously the level of aggregate output at which no additional firm is willing to enter and the behavior of active firms via marginal profits. The following assumption establishes conditions on these effects that yield unambiguous results.

\footnotetext{
${ }^{3}$ It is easy to show that under A $1-5$ such a selection exists

${ }^{4}$ Proof: Let $\left(q^{i}, Q^{i}, n^{i}\right) i=0,1$ be two SCEFE with $n^{1}>n^{0}$ Since $Q^{i}=\left(n^{i}-1\right) R\left(Q^{i}\right), i=0,1$ we obtain that $Q^{1} / R\left(Q^{1}\right)>Q^{0} / R\left(Q^{0}\right)$ which, given that $\mathrm{d} R / \mathrm{d} Q<0$, implies $Q^{1}>Q^{0}$ On the other hand, $\mathrm{d} R / \mathrm{d} Q>-1$ implies that $R\left(Q^{1}\right)-R\left(Q^{0}\right) / Q^{1}-Q^{0}>-1$ Given that $Q^{1}>Q^{0}$, we obtain that $R\left(Q^{1}\right)>R\left(Q^{0}\right)+Q^{0}-Q^{1}$ which, given that $q^{i}=R\left(Q^{i}\right), i=0,1$, implies that $x^{1}>x^{0}$
} 


$$
\text { (A.6) } V_{b}>0, V_{q b} \geq 0 \text {. Moreover } \frac{V_{q b}}{V_{b}} \leq \frac{V_{q Q}}{V_{Q}} \text { in the interior of } \mathbb{R}_{+}^{2} \times B \text {. }
$$

A.6 says two things: (1) $b$ affects positively to both payoffs and marginal payoffs; (2) the effect on marginal profits of a change in $b$ relative to the effect of $b$ on profits cannot be larger than the effect on marginal profits of a change in $Q$ relative to the effect of $Q$ on profits.

In order to understand A.6, let us assume that payoffs are profits, the inverse demand function is $p=e-a q-a Q$ and the variable cost function is $c q+d q^{2} / 2$. Thus, letting $\alpha \equiv e-c$ and $\beta \equiv a+d / 2$,

$$
V=(e-a q-a Q) q-c q-\frac{d}{2} q^{2}=(\alpha-\beta q-a Q) q .
$$

It is easy to see that A.6 holds in the case where the parameter $b$ is $\alpha$, but it does not hold when $b$ is $\beta$.

We now show that under A.6 an increase in $b$ increases the SCEFE values of $n$ and $n \times q$.

Theorem 3. Let $\left(n_{1}, q_{1}\right)=e\left(b_{1}, k\right)$ and $\left(n_{2}, q_{2}\right)=e\left(b_{2}, k\right)$ with $b_{1}>b_{2}$. If A.6 holds,

(i) $n_{1}=n_{2}$ implies $n_{1} q_{1} \geq n_{2} q_{2}$.

(ii) $n_{1} \neq n_{2}$ implies $n_{1}>n_{2}$, and $n_{1} q_{1}>n_{2} q_{2}$.

Proof. (i) In this case the result follows from the fact that our model is identical to the Cournot model with a given number of firms (see, e.g., Corchon, 1994, Proposition 6).

(ii) Recall that $r(Q, b)$ is the best reply correspondence under zero fixed costs. Let $\tilde{Q}(b, k)=\operatorname{Max}\{Q \in \mathfrak{R}: V(r(Q, b), Q, b)=k\}$, and $\tilde{q}(b, k)=r(\tilde{Q}(b, k), b)$. Notice, that if we fix $b, \tilde{Q}(b, k)$ equals $\hat{Q}(k)$ as defined in the proof of Theorem 2 .

Thus, if $(n, q)=e(b, k)$, then $n=\operatorname{Max}\left\{n \in N: n \leq \frac{\tilde{Q}(b, k)}{\tilde{q}(b, k)}+1\right\}$.

Then, $\tilde{Q}_{b}=-\frac{V_{b}}{V_{Q}}>0$ and $\tilde{q}_{b}=\frac{V_{b}}{V_{q q}}\left(\frac{V_{q Q}}{V_{Q}}-\frac{V_{q b}}{V_{b}}\right) \leq 0$ (by A.6). Hence, $n_{1}>n_{2}$.

Now we show that $n_{1} q_{1}>n_{2} q_{2}$. We have $-1<r_{Q}<0$ and $r_{b} \geq 0$. Assume $\left(n_{1}-\right.$ 1) $q_{1} \leq\left(n_{2}-1\right) q_{2}$. Since $n_{1}>n_{2}$, it has to be that $q_{1}<q_{2}$. But

$$
q_{1}=r\left(\left(n_{1}-1\right) q_{1}, b_{1}\right) \geq r\left(\left(n_{2}-1\right) q_{2}, b_{1}\right) \geq r\left(\left(n_{2}-1\right) q_{2}, b_{2}\right)=q_{2}
$$

which is a contradiction. Hence, $\left(n_{1}-1\right) q_{1}>\left(n_{2}-1\right) q_{2}$. Now we have

$$
\begin{aligned}
n_{1} q_{1} & =\left(n_{1}-1\right) q_{1}+r\left(\left(n_{1}-1\right) q_{1}, b_{1}\right)>\left(n_{2}-1\right) q_{2}+r\left(\left(n_{2}-1\right) q_{2}, b_{1}\right) \\
& \geq\left(n_{2}-1\right) q_{2}+r\left(\left(n_{2}-1\right) q_{2}, b_{2}\right)=n_{2} q_{2} .
\end{aligned}
$$

We now present a counterexample to Theorem 3 when A.6 does not hold. 
Example. Let $V=(\alpha-\beta q-a Q) q$, as in the example given after A.6. The best reply function of the representative firm when $k$ is zero is

$$
q=\frac{(\alpha-a Q)}{2 \beta} \text {. }
$$

Let $\alpha, \beta$ and $a$ be functions of $b$. It is easy to compute that,

$$
\tilde{Q}(b, k)=\frac{\alpha}{a}-\frac{2}{a} \sqrt{\beta k} .
$$

Let $\alpha^{\prime}, \beta^{\prime}$ and $a^{\prime}$ be the values of $\alpha, \beta$ and $a$ after the change in $b$. This change is chosen to be such that (see Fig. 1):

1. The slope of the best reply function remains constant, i.e., $a / \beta=a^{\prime} / \beta^{\prime}$.

2. $\tilde{Q}(b, k)$ remains constant, i.e., $\alpha / a-2 / a \sqrt{\beta k}=\alpha^{\prime} / a^{\prime}-2 / a^{\prime} \sqrt{\beta^{\prime} k}$.

3. $R(0, b, k)$ increases, i.e., $\alpha / \beta<\alpha^{\prime} / \beta^{\prime}$.

We will show that such a change is possible: given that, from 1 above, $\beta^{\prime}=a^{\prime} \beta / a, 3$ and 2 above are equivalent to

$$
\frac{a^{\prime} \alpha}{a}<\alpha^{\prime}=\frac{\alpha a^{\prime}}{a}-\frac{2 a^{\prime}}{a} \sqrt{\beta k}+2 \sqrt{\frac{k \beta a^{\prime}}{a}} .
$$

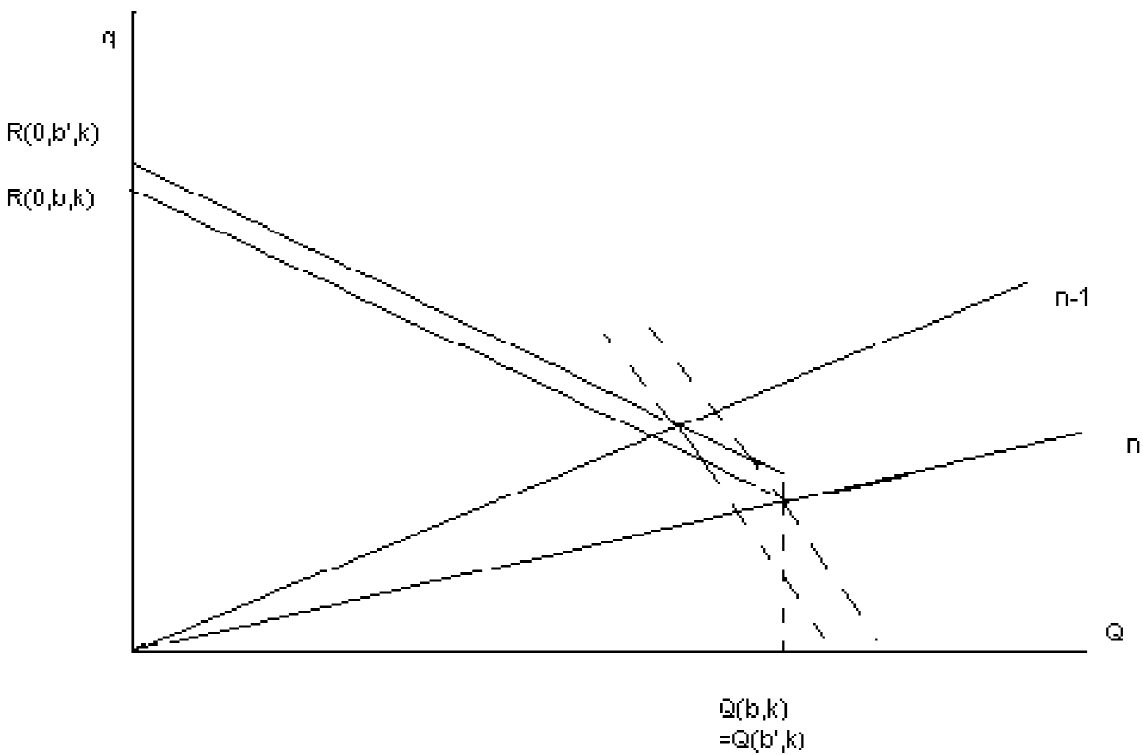

Fig 1 
The interval in which $\alpha^{\prime}$ lies is non-empty if and only if $a>a^{\prime}$. Thus, choose $a>a^{\prime}, \alpha^{\prime}$ belonging to the above interval and $\beta^{\prime}=a^{\prime} \beta / a(<\beta)$.

In Fig. 1 we picture SCEFE before and after the change in $b$. The SCEFE with the maximum number of firms is located in the intersection of the best reply correspondence with the line representing the higher number of firms (e.g., $n$ or $n-1$, etc.). We see that the best reply correspondence after the change in $b$ lies upwards the best reply correspondence before the change in $b$. However, the SCEFE after the change is such that the number of firms decreases. What about aggregate output $q+Q$ ? First notice that $q+Q$ can be measured in Fig. 1: Given any point $\left(q^{\prime}, Q^{\prime}\right)$ all points located on the $45^{\circ}$ line passing through $\left(q^{\prime}, Q^{\prime}\right)$ (the dotted lines in Fig. 1) are such that aggregate output is identical. At any point located to the left of this line aggregate output is less than $q^{\prime}+Q^{\prime}$. We see in Fig. 1 that if $R(0, b, k)$ and $R\left(0, b^{\prime}, k\right)$ are sufficiently close, the intersection of $R\left(, b^{\prime}, k\right)$ with the line $n-1$ is such that aggregate output in the SCEFE relative to $b^{\prime}$ is smaller than the aggregate output in the SCEFE relative to $b$.

The intuition behind this example is that if demand shifts out but is also made more elastic, this intensifies competition in such a way that fewer firms find entry profitable and, as a result of fewer firms, industry supply is reduced in spite of larger demand.

Finally, we check which part of A.6 does not hold. Notice that in the example above, $R$ ( ) shifts upwards. Since $\mathrm{d} q / \mathrm{d} b=-V_{q b} / V_{q q}>0$, it must be that $V_{q b}>0$. Also, since $\tilde{Q}_{b}=-V_{b} / V_{Q}=0$ it must be that $V_{b}=0$. However, the example could have been constructed if $\tilde{Q}$ increases just a little bit (see Fig. 1) and in this case, $V_{b}>0$. However, the last part of A6 is violated because if $V_{q b} / V_{b} \leq V_{q Q} / V_{Q}$, easy calculations show that $\beta^{\prime} \geq \beta$, contradicting that $\beta^{\prime}<\beta$.

\section{Observable regularities}

In this section we will be concerned with the following question. Suppose that some variables (e.g., price, profits, output, etc.) are observable. Does the notion of a SCEFE restrict in any way these observations? In other words, is any observation of these variables compatible with the hypothesis of the model? We will first assume that we have observations about $q$ and $Q$. The question is: Is any set of observations on $q$ and $Q$ compatible with the hypothesis that these observations are on the best reply correspondence of the representative firm? In order to answer this question let us introduce the following concept.

Definition 2. A correspondence $f: \mathbb{R}_{+} \rightarrow \mathbb{R}_{+}$is said to be admissible if:

(i) $\forall Q \in[0, y)$, some $y>0, f$ is $C^{o}$ with positive values and strictly decreasing.

(ii) When $Q=y, f$ has two values namely $\lim _{z \rightarrow y} f(z)$ with $z \in[0, y)$ and 0 .

(iii) For $Q>y, f(Q)=0$.

(iv) $\partial f / \partial Q>-1$ whenever $f \in C^{1}$.

The following result shows that, under A.1-5, admissible and best reply correspondences, are equivalent concepts. 
Theorem 4. (a) Under A.1-5, the best reply correspondence $R$ is admissible. (b) Let $f$ be an admissible correspondence. There exists a cost function $C$, which is $C^{1}$ in $\mathbb{R}_{++}$, and a linear inverse demand function $p=A-q-Q$ such that

$$
\text { if } \pi(q, Q) \equiv(A-q-Q) q-C(q) \text {, then } f(Q) \equiv \arg \max _{q \in \Re_{+}}(A-q-Q) q-C(q) \text {. }
$$

where $\pi$, as defined above, fulfills A.1-5.

Proof. Part (a) follows easily (see our comments to A.1 - 5 above).

(i) $R>0$ in $[0, y)$ follows from the second part of A.1, and $\mathrm{d} R / \mathrm{d} Q<0$. Notice that $y=\tilde{Q}(b, k)$.

(ii) follows from $0 \notin K$, and A.3-4.

(iii) follows from $0 \notin K$, and A.2-3-4.

(iv) follows from $0>V_{q Q}>V_{q q}$.

Let us now prove part (b). let $\bar{Q}=y+a, a>0$. Let $F$ be a continuous extension of $f$ to $[0, \bar{Q}]$ with $F$ strictly decreasing and $F(\bar{Q})=0 . F$ is invertible. Since $F \in C^{o}$, $F^{-1} \in C^{o}$. Also, $F^{-1}$ is bounded, and, thus, $F^{-1}$ is integrable (see Bartle, 1976, pp. 156, 427). Let $t(q)$ be the primitive of $F^{-1}(q)$ for $q \in[0, f(0)] . t$ can be extended to $\mathbb{R}_{+}$such that $t \in C^{o}$ for $q>f(0)$. By choosing appropriately the integration constant $t(0)=0$. Let

$$
C(q) \equiv A q-q^{2}-t(q)+k \text { if } q>0 \text { and } C(0)=0 \text { if } q=0,
$$

where $k$ is a positive number to be determined later on. Take $A$ such that $C(q)>0$ and $\mathrm{d} C / \mathrm{d} q>0, \forall q \in[0, f(0)]$. Let $p=A-q-Q$. Define:

$$
\begin{aligned}
& \pi \equiv p(x) q-C(q)=(A-q-Q) q-A q+q^{2}+t(q)-k . \\
& \text { Thus, } \frac{\partial \pi}{\partial q}=F^{-1}(q)-Q=0 .
\end{aligned}
$$

Since $F^{-1}$ is strictly decreasing, second-order conditions of profit maximization are satisfied and thus, $F^{-1}(q)=Q$ determines a profit maximizing output if $k=0$. Since $F^{-1}$ is strictly decreasing beyond $f(0)$, outputs greater than $f(0)$ are never chosen. Therefore, $F^{-1}(q)=f^{-1}(q)$ and thus, $q=f(Q)$. Now let $k=t(f(y))-y f(y)$ with $y$ as defined in Definition 2, part ii. Thus

$$
\pi=t(q)-Q q-k, \text { if } q>0 .
$$

If $Q=y$, the firm is indifferent between producing 0 and $f(y)$. Notice that $\mathrm{d} \pi / \mathrm{d} Q=$ $t^{\prime} f^{\prime}-f^{\prime} Q-f=-f<0$ and thus payoffs are positive for $Q<y$. By the same token, payoffs would be negative if the firm produced a positive output for $Q>y$. Thus, $f$ is the best reply correspondence of a firm with payoff function $\pi$. We will show now that $\pi$ fulfills A.1-5.

A.1: Since at $Q=0$, the firm chooses positive output and $\pi$ is strictly concave on $q, k$ (as defined above) $>0$ and $\max _{q} \pi(q, 0)>0$.

A.2: It follows from the definition of $\pi$.

A.3: From the definition of $t$ is clear that $\pi<0$ for $q$ large enough.

A.4: It follows from the previous arguments

A.5: It follows from the definition of $\pi$ and $F$. 
Next, we will concentrate on the following question. Suppose we have an observation about prices, profits and number of active firms. Is there any relationship between these variables, for instance between profits and the number of active firms, were they generated by a SCEFE? The answer is no, even under strong functional restrictions on the shape of demand and costs.

Theorem 5. Let $(\tilde{n}, \tilde{p}, \tilde{\pi}) \in \mathbb{N} \times \mathbb{R}_{+}^{2}$ be an observation of a number of firms $(\tilde{n}>1)$, a price and profits. There is an isoelastic demand function with unit elasticity and a cost function with constant marginal costs for which the SCEFE with maximal number of firms is $(\tilde{n}, \tilde{p}, \tilde{\pi})$.

Proof. Let $x$ be aggregate output, i.e., $x=q+Q$. Let $p=A / x$ be the candidate inverse demand function and let $C_{i}=c q+k$ (if $q>0$ ) and $C_{i}=0$ (if $q=0$ ) be the candidate cost function. Given these two functions a necessary and sufficient condition for $(p, n, \pi)$ to be a SCEFE with maximal number of firms is that

$$
p=\frac{c n}{n-1}, n=E\left[\sqrt{\frac{A}{k}}\right], \pi=\frac{A}{n^{2}}-k, \quad \text { where } E(w) \equiv \text { integer part of } w .
$$

We will now construct an inverse demand and a cost function, i.e., we will find values of the parameters $A, k$ and $c$ such that the SCEFE of this economy is $(\tilde{n}, \tilde{p}, \tilde{\pi})$. Let

$$
A=\frac{(\tilde{n}+\varepsilon)^{2} \tilde{\pi} \tilde{n}^{2}}{\epsilon^{2}+2 \epsilon \tilde{n}}, k=\frac{\tilde{\pi} \tilde{n}^{2}}{\epsilon^{2}+2 \epsilon \tilde{n}}, c=\frac{\tilde{p}(\tilde{n}-1)}{\tilde{n}},
$$

where $\epsilon$ is an arbitrary number in $(0,1)$. The SCEFE for this economy is:

$$
p=\frac{\tilde{p}(\tilde{n}-1)}{\tilde{n}} \cdot \frac{\tilde{n}}{(\tilde{n}-1)}=\tilde{p}, \quad \pi=\frac{(\tilde{n}+\varepsilon)^{2} \tilde{\pi}}{\epsilon^{2}+2 \epsilon \tilde{n}}-k=\tilde{\pi}, n=E[\tilde{n}+\epsilon]=\tilde{n} .
$$

Thus, the proposition is proved.

Suppose now that the fixed cost $k$ is also observable. Does the previous result change? The answer is, not much.

Theorem 6. Let $(\tilde{n}, \tilde{p}, \tilde{\pi}, \tilde{k}) \in \mathbb{N} \times \mathbb{R}_{+}^{3}$ be an observation of a number of firms $(\tilde{n}>1)$, a price, profits and fixed costs, such that $\tilde{\pi} / \tilde{k}<1 / \tilde{n}^{2}+2 / \tilde{n}$. There is an isoelastic demand curve with unit elasticity and a cost function with constant marginal costs and a fixed costs $\tilde{k}$ for which the SCEFE equilibrium with maximal number of firms is $(\tilde{n}, \tilde{p}, \tilde{\pi})$. 
Proof. As before take $\tilde{k}=\tilde{\pi} \tilde{n}^{2} /\left(\epsilon^{2}+2 \epsilon \tilde{n}\right)$. Solving this equation we obtain that

$$
\epsilon=-\tilde{n}+\sqrt{\tilde{n}^{2}+\frac{\tilde{\pi} \tilde{n}^{2}}{\tilde{k}}}(>0) \text {. Thus } \epsilon<1 \Leftrightarrow \frac{\tilde{\pi}}{\tilde{k}}<\frac{1}{\tilde{n}^{2}}+\frac{2}{\tilde{n}} .
$$

Similar calculations to the one performed in the proof of Theorem 5 show that the required parameters are:

$$
A=\frac{(\tilde{n}+\varepsilon)^{2} \tilde{\pi} \tilde{n}^{2}}{\epsilon^{2}+2 \epsilon \tilde{n}}, \quad c=\frac{\tilde{p}(\tilde{n}-1)}{\tilde{n}}, \quad \epsilon=-\tilde{n}+\sqrt{\tilde{n}^{2}+\frac{\tilde{\pi} \tilde{n}^{2}}{\tilde{k}}} .
$$

Notice that because $0<\epsilon<1, E[\tilde{n}+\epsilon]=\tilde{n}$. Thus, the proposition is proved.

Similar results can be obtained by assuming that the inverse demand is linear. In this case a similar reasoning to the one used above shows that the bound on the rate of profits is

$$
\frac{2 \tilde{n}+3}{\tilde{n}^{2}+2 \tilde{n}+1}, \text { less than } \frac{1}{\tilde{n}^{2}}+\frac{2}{\tilde{n}},
$$

but still substantial:

$$
\text { If } \tilde{n}=20, \frac{2 \tilde{n}+3}{\tilde{n}^{2}+2 \tilde{n}+1} \simeq 9 \%,
$$

not far for the $10 \%$ obtained in the isoelastic case.

Summing up, Theorems 5 and 6 make the point that SCEFE predicts very little about the relationship between concentration and profitability.

\section{Conclusions}

In this paper we have studied the properties of the Cournot model with free entry. Two things are worth noticing:

1. Comparative statics may yield different answers from those obtained in the Cournot model with a given number of firms. In particular, the shift of a parameter affecting positively both payoffs and marginal payoffs may decrease aggregate output due to a fall in the number of active firms.

2. The model studied here does not support the view that high profit rates can only be sustained by preventing entry or by collusion of existing firms. Even if fixed costs are observable, the model does not predict a straight relationship between number of firms and profit rates.

Notice that the facts noticed in Points (1) and (2) cannot occur in perfectly competitive markets. The fact that we have selected the SCEFE with maximal number of firms, makes our results stronger because they occur in the allocation which is the closest that we can get to the perfectly competitive allocation. 
We hope that future research will investigate if Points (1) and (2) above arise in other models of imperfectly competitive markets with endogenous number of firms, e.g., in models of product differentiation.

\section{Acknowledgements}

We are grateful to C. Beviá, R. Faulí-Oller, P. Marín, A. Manelli, D. Pérez-Castrillo, M. Pastor, an associate editor and a referee for very good suggestions. This research was financed by CICYTs PB98-0024 and PB98-0867 and by DURSI 20011 SGR00162.

\section{References}

Bartle, R G, 1976 In: The Elements of Real Analysis, 2nd Edition Wiley, New York

Bain, J, 1956 In: Barriers to New Competition Harvard University Press, Cambridge, MA

Bulow, J , Geanakoplos, J , Klemperer, P, 1985 Multimarket oligopoly: strategic substitutes and complements Journal of Political Economy June, 488-511

Cabral, L, 1997 Entry Mistakes, CEPR Discussion paper No 1729

Cabral, L, 2000 In: Introduction to Industrial Organization MIT Press, Cambridge, MA

Carlton, D, Perloff, J, 1990 In: Modern Industrial Organization Harper Collins, New York

Corchon, L, 1994 Comparative statics for aggregative games: The strong concavity case Mathematical Social Sciences 28, 151-165

Corchon, L, 1996 In: The Theories of Imperfectly Competitive Markets, 2nd Edition Lecture Notes in Economics and Mathematical Systems, Vol 442 Springer, Berlin, Hard cover 2000

Corchon, L, Fradera, I, 1996 Comparative Statics in Free Entry Cournot Equilibrium, Working Paper, Universitat Autonoma de Barcelona

Dixit, A, 1986 Comparative statics for oligopoly International Economic Review February, 107-122

Friedman, J, 1982 Oligopoly Theory In: Arrow, K, Intrilligator, M (Eds ) Handbook of Mathematical Economics North Holland, Amsterdam, Chapter 11

Frank, C R , 1965 Entry in a Cournot market Review of Economic Studies 32, 245-250

Hahn, F H , 1962 The stability of Cournot Oligopoly Solution Review of Economics Studies 29, 329-333

Lippman, S A, Mamer, JW, McCardle, KF, 1987 Comparative statics in non-cooperative games via transinfinitely iterated play Journal of Economic Theory 41, 288-303

Lopez-Cuñat, J , 1997 One-stage and Two-stage Entry Cournot Equilibria Mimeo, U de Alicante

Martin, M , 1993 In: Advanced Industrial Economics Blackwell, Cambridge, MA

McManus, M , 1962 Numbers and size in Cournot oligopoly Yorkshire Bulletin 14, 14-22

McManus, M , 1964 Equilibrium, numbers and size in Cournot oligopoly Yorkshire Bulletin 16, 68-75

Milgrom, P, Roberts, J , 1990 Rationalizability, learning and equilibrium in games with strategic complementarities Econometrica 58 (6), 1255-1277

Milgrom, P, Shannon, C, 1992 Monotone Comparative Statics Mimeo Stanford University

Novshek, W, 1980 Cournot equilibrium with free entry Review of Economic Studies 47, 473-486

Okuguchi, K, 1973 Quasi-competitiveness and Cournot oligopoly Review of Economic Studies 40, 145-148

Quirmbach, H, 1988 Comparative Statics for Oligopoly Demand Shift Effects, International Economic Review August, 451-459

Ruffin, R J , 1971 Cournot oligopoly and competitive behavior Review of Economic Studies 38, 493-502

Seade, J , 1980 On the effects of entry Econometrica 48 (2), 256-278

Szidarovsky, F, Yakowitz, S , 1982 Contributions to Cournot oligopoly theory Journal of Economic Theory $28,51-70$ 
Vives, X., 1990. Nash equilibrium with strategic complementarities. Journal of Mathematical Economics 19, 305-321.

Yarrow, G.K., 1985. Welfare losses in oligopoly and monopolistic competition. The Journal of Industrial Economics XXXIII (4), 515-529. 